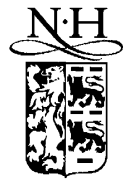

ELSEVIER

\title{
Interband phonon assisted tunneling in InAs/GaSb heterostructures
}

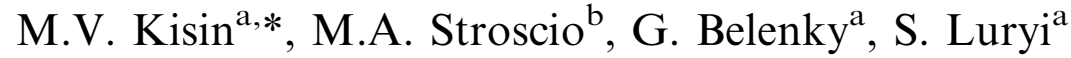 \\ ${ }^{a}$ Department of Electrical and Computer Engineering, SUNY at Stony Brook, NY 11794-2350, USA \\ ${ }^{\mathrm{b}}$ US Army Research Office, P.O. Box 12211, Research Triangle Park, NC 27709, USA
}

\begin{abstract}
The rate of LO-phonon assisted interband transitions in an InAs/GaSb double quantum well heterostructure is compared with the elastic interband tunneling rate through the heterostructure 'leaky window'. We show that the phonon-assisted process can dominate over the elastic tunneling if the initial and final electron states anticross and the anticrossing gap is smaller than the LO-phonon energy. (C) 2002 Elsevier Science B.V. All rights reserved.
\end{abstract}

Keywords: Type-II heterostructures; LO-phonon emission; Interband transitions

Electron transitions assisted with elastic interband tunneling and/or LO-phonon emission are of vital importance for intersubband type-II cascade lasers, where they determine the depopulation of the lower lasing states mostly localized in electrontype active quantum well (QW); see the band diagram in the inset of Fig. 4, level L1-. The rate of the interband tunneling, $\Gamma_{\text {tun }}$, was studied in our previous paper [1] for a model type-II double quantum well (DQW) InAs/GaSb heterostructure. In interband tunneling process, electron, after the intersubband $\mathrm{L} 2 \rightarrow \mathrm{L} 1-$ light-emitting transition, can transit with the same energy through the heterostructure 'leaky window', which is the energy range above the InAs conduction and below the GaSb valence bands. Final states for this interband tunneling belong to emitter/collector region, which in our model is represented by InAs half-space layer. In this work we calculate the rate of the L1- level depopulation due to LO-

\footnotetext{
*Corresponding author. Fax: + 1-631-632-8494.

E-mail address: mvk@ece.sunysb.edu (M.V. Kisin).
}

phonon emission process, $\Gamma_{\mathrm{ph}}$, and compare it with the tunneling rate $\Gamma_{\text {tun }}$. Initial and final electron states involved in the phonon-emission process belong, correspondingly, to the lowest electronlike and the highest light-hole-like subbands located in the upper part of the leaky window. In analogy with the subband depopulation in type-I DQW heterostructures [2], we can expect that anticrossing between these two subbands will result in efficient electron-phonon overlap and, consequently, will circumvent the symmetry constraint for the phonon-assisted interband transitions characteristic of type-I tunneling heterostructures [3]. In the chosen range of the QW widths, the anticrossing gap is very close to LOphonon energy in constituent semiconductors, $n \omega_{\text {LO }} \approx 30 \mathrm{meV}$; see Fig. 1 . Therefore, LO-phonon emission is a nearly resonant process with a small momentum transfer and a high characteristic rate, so that the elastic interband tunneling from the upper of the anticrossing levels, L1-, can be significantly enhanced by the LO-phonon assisted depopulation through the lower lying level, L1 + . 


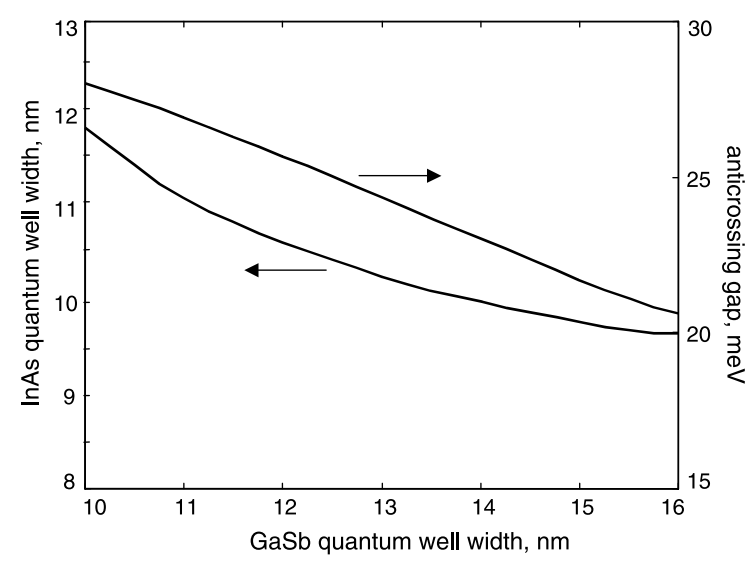

Fig. 1. Relationship between InAs and GaSb QW widths, which provides for the anticrossing between the lowest electronlike and the highest light-hole-like levels. The upper curve shows the anticrossing gap at electron momentum $K=0$.

Inasmuch as the polar mode confinement has not yet been studied in the InAs/GaSb heterosystem, we calculate the phonon emission rate using a model bulk-like phonon spectrum. For illustrative purpose, we consider also confined LOphonon modes of InAs and GaSb layers, which are of primary importance for phonon-assisted interwell transitions in type-I DQW heterostructures; see Ref. [2]. Fig. 2 shows the electron-phonon coupling coefficients, which are mostly determined by corresponding overlap integrals [4], as function of the phonon wave number $q$ for InAs and GaSb constituent materials. All the dependencies have pronounced maximums, firstly, because of drastic decrease in electron-phonon overlap integral at zero momentum transfer $q \sim 0$ due to orthogonality of initial and final electron states in 'vertical' transitions, and, secondly, because of corresponding decrease of the overlap integral at large phonon wave numbers $q>0.2 \mathrm{~nm}^{-1}$. Fig. 2 shows also the total of the coupling coefficients for confined modes in both A- and B-layers (dashed line). At larger wave numbers $q$, in full agreement with the sum rule for electron-phonon interaction, the total of the coupling constants for confined modes fall into the gap between the corresponding parameters of constitutive materials; see Ref. [4]. The inset in Fig. 2 shows the electron-phonon overlap integrals for InAs (curve A) and $\mathrm{GaSb}$

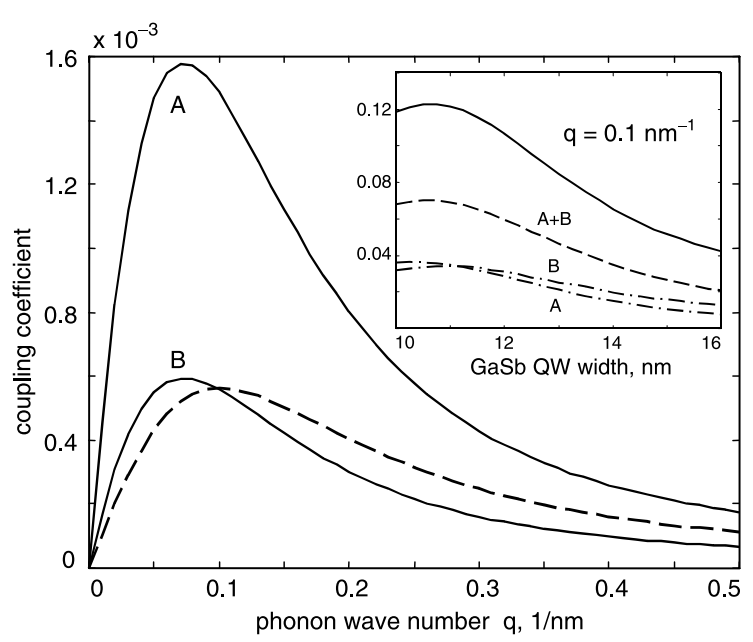

Fig. 2. Electron-phonon coupling coefficients for InAs (A) and GaSb (B) bulk-like phonons (solid lines) and total of coupling coefficients for LO-confined modes (dashed line). The inset shows the overlap integrals at the anticrossing point as function of GaSb QW width for LO-confined phonons in A and B layers (dashed-dotted lines) and their sum (dashed line) in comparison with the overlap integral for bulk-like phonons (solid line).

(curve B) LO-confined phonon modes and compares their sum (dashed line) with the overlap integral in the quasibulk approximation (solid line). Since the phonon wave number in this exemplary calculation is relatively small $\left(q=0.1 \mathrm{~nm}^{-1}\right)$, there is a significant difference between the two latter curves, which, according to the sum rule, can be attributed to the possible existence of the interface phonon modes in this system. The influence of the interface modes should decrease with the increasing phonon wave number $q$. This indeed is readily seen from Fig. 2 .

To estimate the resonant phonon emission rate, we consider here only the most important electron transitions with small momentum transfer. At the resonance, the emission rate is very sensitive to the fine structure of the electron energy spectrum, particularly, to the density of final states for electron transition. In an asymmetric heterostructure, the subband dispersion contains linear terms, $\pm \alpha K$, which dominate the dispersion and determine the density of states for small electron momenta $K$; see also Ref. [1]. These terms describe the splitting of otherwise doubly degenerate 


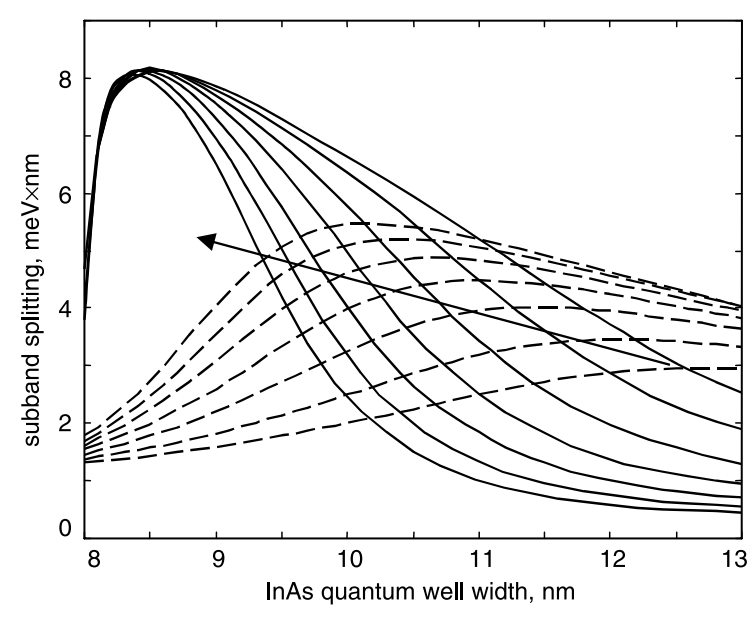

Fig. 3. Splitting parameter $\alpha$ for L1- (solid lines) and L1+ (dashed lines) subbands in InAs/GaSb DQW heterostructures. The curves of each set in the direction of the arrow correspond to the GaSb QW width increasing from 10 to $16 \mathrm{~nm}$ in $1 \mathrm{~nm}$ increment.

subbands. Fig. 3 shows the splitting parameter $\alpha$ for initial (solid lines) and final (dashed lines) electron states participating in the phonon emission process. Under the anticrossing condition, the splitting parameter $\alpha$ is equal for initial and final electron states. The relatively small subband dispersion at small $K$ results in a high density of the final electron states and leads to a high resonant phonon emission rate. Fig. 4 (solid lines) shows the rate $\Gamma_{\mathrm{ph}}$ of the LO-phonon assisted electron transitions. Upper (A) and lower (B) curves correspond to the emission rate for bulklike InAs and GaSb LO-phonons, respectively. The dashed line shows the total depopulation rate due to the emission of the confined phonons in the InAs and GaSb QW layers. All these rates demonstrate significant increase in the vicinity of the phonon resonance, when the anticrossing gap approaches the LO-phonon energy (narrow GaSb QW; see Fig. 1). Though the highest possible rates are characteristic for only a small fraction of initial and final electron states involved in the transitions

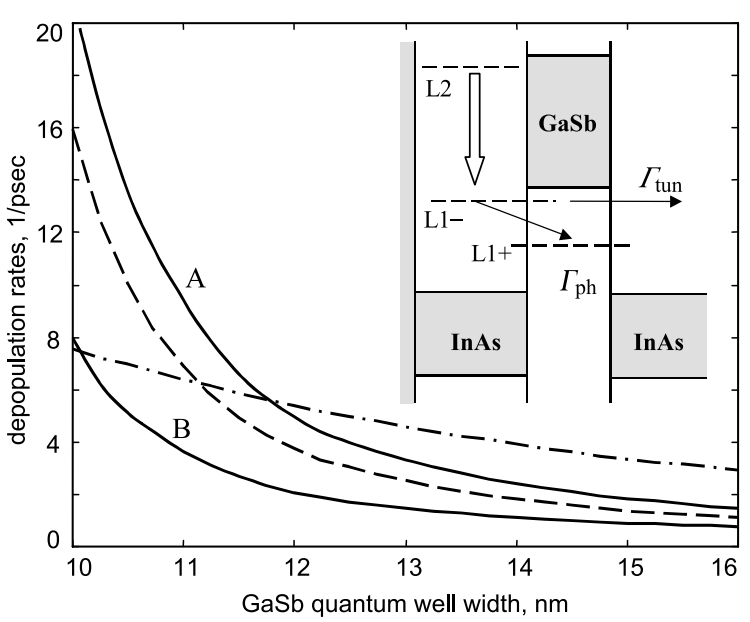

Fig. 4. Depopulation rate of the lowest lasing level (L1-). Lasing transition is shown by the bold arrow. Spontaneous LOphonon emission rates for the bulk-like InAs $\left(\Gamma_{\mathrm{phA}}\right)$ and $\mathrm{GaSb}$ $\left(\Gamma_{\mathrm{phB}}\right)$ phonons are shown by solid lines. The dashed line shows the total of the confined phonon emission rates in the InAs and GaSb QW layers. Elastic interband tunneling rate $\Gamma_{\text {tun }}$ is shown by dashed-dotted line.

with small momentum transfer, the overall typical rates represented in Fig. 4 demonstrate high efficiency of the LO-phonon assisted depopulation. At resonance, $\Gamma_{\mathrm{ph}}$ can substantially exceed the rate of the direct interband tunneling $\Gamma_{\text {tun }}$.

This work was supported by ARO grant DAAD 190010423 and AFOSR Grant F49620-00-10331.

\section{References}

[1] M.V. Kisin, M.A. Stroscio, S. Luryi, G. Belenky, Physica E 10 (2001) 576.

[2] M.A. Stroscio, M.V. Kisin, G. Belenky, S. Luryi, Appl. Phys. Lett. 75 (1999) 3258.

[3] Yu.B. Lyanda-Geller, J.-P. Leburton, Appl. Phys. Lett. 67 (1995) 1423.

[4] M.V. Kisin, M.A. Stroscio, G. Belenky, V.B. Gorfinkel, S. Luryi, J. Appl. Phys. 83 (1998) 4816. 